\title{
Desafíos del desarrollo de guías de práctica clínica (GPC) para el cáncer pediátrico en Colombia
}

\author{
A. Linares, M. Torres, R. Pardo, M. Vizcaíno, I. Sarmiento, O. Ramírez, F. Molina
}

Universidad Nacional de Colombia, Alianza Cinets.

Objetivo. La necesidad de estandarizar la gestión de las neoplasias hematológicas y llegar a las mismas tasas de supervivencia de los países desarrollados requiere la elaboración de guías de práctica clínica (GPC) en nuestro país. La supervivencia global de la leucemia linfoide aguda en Colombia es alrededor del 50\%, mientras que en otros países es cerca de un $80 \%$.

Material y métodos. El Ministerio de Salud y Protección Social asignó el desarrollo de una GPC de leucemias agudas y linfomas en pediatría. El grupo de la guía (GDG) enfrentó desafíos por cantidad/calidad de las pruebas y los 'puntos de vista' de los pacientes.

Resultados. Durante la formulación de las recomendaciones, no solo la eficacia es un resultado crítico, otros aspectos, como toxicidad del tratamiento y los efectos adversos, deben ser evaluados. Con el fin de identificar la información relevante, el GDG debía incluir estudios observacionales que conllevaron algunos retos. Se desarrolló un ejercicio cualitativo para la identificación del punto de vista de los pacientes, que fue ponderado con los riesgos y beneficios de las intervenciones.

Conclusiones. Una vez finalizada la guía, las recomendaciones fueron conducidas hacia la seguridad del paciente y la aplicación implícita de realizar cambios en las políticas públicas, mejorar el acceso a la terapia continua, el diagnóstico adecuado y el trabajo en torno a los tratamientos disponibles, para mejorar la supervivencia global.

\section{Trasplante autólogo en niños con enfermedad de Hogdkin. Experiencia de un centro en Colombia}

\author{
- A. Linares, E. Pedraza, V. Abello, C. Rosales, M. Rosales, L. Villamizar, H. Esguerra
}

Unidad de Trasplante, Clínica de Marly.

Objetivo. Evaluar los resultados del trasplante autólogo en niños con enfermedad de Hodgkin (EH) refractaria o en recaída, en un solo centro en Bogotá

Metodología. Treinta niños con EH refractaria o en recaída fueron llevados a trasplante autólogo entre 1998 y 2011. Los aspectos clínicos de la enfermedad previos al trasplante fueron analizados, así como los eventos recaída y muerte postrasplante, para establecer la probabilidad de supervivencia libre de evento (SLE) y supervivencia global (SG) a cinco años.

Resultados. Con una mediana de seguimiento de 34,9 meses, un rango entre 5 y 90 meses, la probabilidad de SG fue del $83,3 \%$ y la SLE fue del 76,6\% a cinco años. La mortalidad relacionada con el trasplante fue del 3,3\%. No se presentaron muertes tóxicas más allá del día 100.

Conclusiones. Este es el primer informe en Colombia de los resultados del trasplante autólogo en niños con EH. Los resultados de este grupo de pacientes son similares a los resultados de otros grupos en países desarrollados. Más de la mitad de los niños con EH refractaria o en recaída pueden ser curados con el trasplante autólogo de precursores hematopoyéticos en nuestro país 